\section{IMPACTOS DE LA \\ REPROGENÉTICA EN LAS \\ TENDENCIAS DEMOGRÁFICAS \\ Y EN LAS ESTRUCTURAS \\ FAMILIARES}

\author{
IMPACTS OF THE \\ REPROGENETICS IN BOTH THE \\ DEMOGRAPHIC TENDENCIES \\ AND THE FAMILY STRUCTURES
}

\author{
Hilde Sánchez Morales \\ UNED \\ Grupo Estudio Tendencias Sociales
}

\begin{abstract}
This paper introduces the family and demographic impact of assisted reproduction and genetic engineering in humans (reprogenetics). We are situated in a complex issue due to the rapid pace at which progress is happening and the lights and shadows associated with its development. The questions we will try to analyze over the next few pages are: How far the family as a "primary group" based on the biological relationship among its specific features -socially regulated-human reproduction, breeding, location and education of children, will be structurally and sociologically modified due to the development of alternatives to the time of descent?, to what extent is affecting reprogenetics Demography as a scientific discipline, and how likely will grow by near future?
\end{abstract}

KEY WORDS: Reprogenetics and Family; reprogenetics and Demography; social impacts of human genetics; social impacts of assisted reproduction.

\section{INTRODUCCIÓN}

Las técnicas de reproducción humana asistida nos sitúan ante procedimientos como la inseminación artificial, la fecundación in vitro, la transferencia intratúbica de gametos, la transferencia intratúbica de zigotos, la inyección intracitoplásmica de espermatozoides, sus derivaciones $y$, eventualmente, a medio plazo, la clonación en sus diversas variantes, la "fecundación sintética", los cultivos de células madre embrionarias, la selección celular inmunomagnática (MACS), etc.

Dichas técnicas están siendo aplicadas desde hace varias décadas en los países más desarrollados ${ }^{1}$ y se encuentran estrechamente relacionadas con los problemas de esterili$\mathrm{dad}^{2}$, evidenciándose desde hace años sus impactos sobre la demográfica y la familia (vid. Cuadro 1).
RESUMEN: En este texto se presentan los impactos familiares y demográficos de la reproducción asistida y la ingeniería genética en humanos (Reprogenética). Nos sitúa en un tema complejo, debido al vertiginoso ritmo con el que se suceden los avances y las luces y sombras asociadas a su desenvolvimiento. Las cuestiones que trataremos de analizar a lo largo de las próximas páginas son las siguientes: ¿Hasta dónde la familia, como "grupo primario" fundamentado en la relación biológica que tiene entre sus funciones especificas -reguladas socialmente- la reproducción humana, la crianza, la ubicación y educación de los hijos, se verá estructural y sociológicamente modificada debido al desarrollo de formas alternativas a la hora de descendencia?, ¿en qué medida la Reprogenética está afectando a la Demografía, como disciplina científica, y cómo previsiblemente lo hará en un futuro cercano?

PALABRAS CLAVE: Reprogenética y familia; Reprogenética y Demografía; impactos sociales genética humana; impactos sociales reproducción asistida.

No en vano los problemas de infertilidad-esterilidad han aumentado en las últimas décadas, constatándose, tanto por parte de la comunidad especializada en reproducción, como por la opinión pública española ${ }^{3}$ (Fundación Sistema, 2000) y expertos en familia (Sánchez Morales, 2001: 66), que tenderán a incrementarse en los próximos años (GETS, 1997; GETS, 2002, y GETS, 2005). Las razones apuntan, fundamentalmente, hacia condicionantes sociales, asociadas a los efectos de los nuevos estilos de vida, y al aumento de la edad media en la que las mujeres tienen sus hijos (INE, varios años) $)^{4}$. Prueba de ello es que, según datos de la Sociedad Española de Fertilidad, aproximadamente el 80\% de las pacientes que siguieron un tratamiento de Fecundación asistida [Fecundación in vitro (FIV) y/o Inyección Intracitoplásmica de Espermatozoides (ICSI o FIV+ICSI)] en el año 2008 tenían una edad inferior a los 40 años (SEF, 2010). 


\begin{tabular}{|l|l|}
\hline \multicolumn{1}{|c|}{ Impactos sobre la demografía } & \multicolumn{1}{c|}{ Impactos sobre la familia } \\
\hline - Sobre la tasa de fertilidad. & Alteran la especificidad de la familia tradicional como "comunidad de sangre". \\
\hline -Sobre la tasa de natalidad. & - Implicación en la familia de actores anónimos (donantes). \\
\hline -Sobre la tasa de mortalidad. & - Personas en estado de vida latente (congelación de embriones). \\
\hline $\begin{array}{l}\text {-Sobre la esperanza media de vida } \\
\text { en los paises desarrollados. }\end{array}$ & $\begin{array}{l}\text { - Impactos sobre la concepción tradicional de maternidad, paternidad, } \\
\text { las estructuras del parentesco y de la propia familia tradicional. }\end{array}$ \\
\hline -Sobre la pirámide de población y su distribución por sexos. & $\begin{array}{l}\text { - Confusionismo derivado de la aparición de nuevas figuras de madres y padres } \\
\text { (biológica, legal, social). } \\
\text { - Nuevos modelos de filiación familiar. } \\
\text { - Familias técnicamente "clonadas". } \\
- \text { Incorporación de criterios de calidad biológica. } \\
\text { - Consolidación del consejo genético familiar. } \\
\text { - Estigmatización familiar en función de criterios genéticos. } \\
\text { - Eugenesia. } \\
\text { - Problemas juridicos derivados de diagnósticos genéticos erróneos. } \\
\text { - Problemas patrimoniales. }\end{array}$ \\
\hline
\end{tabular}

Fuente: Elaboración propia.

Los impactos de la ingeniería genética sobre la familia y la demografía, que aqui vamos a analizar, los centramos fundamentalmente en los efectos derivados de la utilización de los diagnósticos genéticos (diagnósticos preimplacionales/fetales y parentales). Dichos diagnósticos pueden utilizarse en contextos clínicos y no clínicos. En contextos clínicos se dan dos modalidades: diagnósticos prenatales (en embriones o fetos) y diagnósticos parentales (individuos o parejas adultas); y dos variantes, en función de si se intenta diagnosticar una enfermedad hereditaria o una predisposición o susceptibilidad a desarrollar alguna enfermedad. En contextos no clínicos, son utilizados, por ejemplo, en el ámbito laboral, por parte de aseguradoras, compañías médicas y entidades bancarias. La finalidad es este caso es "disminuir" los componentes de riesgo o incertidumbre a la hora de hacer un seguro, conceder un crédito o proporcionar un trabajo.

Los diagnósticos prenatales se realizan protocolariamente en los procesos de fecundación in vitro (diagnósticos preimplantacionales), habiéndose consolidado como una técnica de "control de calidad en los nacidos" en los procedimientos de reproducción asistida. Se analiza el cariotipo del embrión, informándose a la pareja sobre su viabilidad y "salud genotípica/fenotípica". La pareja/ mujer decide si desea le sea implantado 0 , dentro de las posibilidades que ofrece la Ley 14/2006, almacenarlos para futuras transferencias o donarlos a otras parejas. Los diagnósticos prenatales se realizan también en fetos. La Sanidad Pública española los recomienda a las mujeres a partir de los 35 años, siendo la técnica más habitual la amniocentesis.

De hecho, desde que en el año 1996 se realizó el Primer estudio Delphi sobre Biogenética en los sucesivos estudios posteriores, así como en el Estudio Delphi sobre Tendencias familiares en España (Sánchez Morales, 2001) del GETS del año 2000, los expertos han ido formulando las previsiones de que en los países tecnológicamente más avanzados se produciría una reducción de las muertes fetales y de los nacimientos de bebés prematuros por sistemas de control de información del fetos. Igualmente se estimó que hacia el 2010 nacerian menos niños con enfermedades genéticas, habiendo contribuido, desde nuestra perspectiva, la difusión de estas pruebas diagnósticas. Las tendencias de los últimos años han confirmado estas hipótesis ${ }^{5}$. 
En lo que a los diagnósticos parentales se refiere destacar la situación en la que quedaría una persona a la que se determina que va a padecer una predisposición o una probabilidad muy elevada de sufrir una enfermedad para la que no existen alternativas terapéuticas (diagnóstico parental). También su familia quedaría afectada al compartir el mismo acervo genético, haciendo su aparición un tipo de familia "sana-enferma", con los efectos discriminatorios que ello tendría. Asimismo, deben valorarse las implicaciones derivadas si esta información, de carácter confidencial que afecta únicamente a los actores involucrados, se deslizara hacia contextos no clínicos. Cabría introducir un elemento de estigmatización social, que llevaría a las personas desfavorablemente diagnosticadas y a sus familias a situaciones de vulnerabilidad a la hora, por ejemplo, de acceder a un puesto de trabajo, de contratar una póliza médica o de seguros, sin minusvalorar los efectos psicológicos causados en los así diagnosticados.

Otro tipo de diagnóstico parental es el que se proporciona a mujeres/parejas de "riesgo" que desean asesorarse a la hora de tener descendencia y conforme al resultado deciden tener o no tener hijos ${ }^{6}$.

Si hay una disciplina científica donde los avances en $R e-$ progenética han repercutido es sobre la Demografía y si hay un grupo que se ha visto concernido más directamente es la familia. De hecho han sido afectados indicadores como la tasa de fertilidad, la tasa de natalidad, la tasa de mortalidad y la esperanza media de vida. Por lo que resulta necesario adecuarlos a las nuevas realidades y avanzar en la conceptualización de la Demografía como área de conocimiento.

Por otro lado, la Reprogenética altera la especificidad tradicional de la familia de constituir una "comunidad de sangre", pues los límites biológicos del grupo familiar se pueden traspasar, dándose entrada a otros participantes que nunca llegarán a ser miembros del núcleo familiar -y ni serán conocidos- pero que adquieren un protagonismo clave en todo el proceso ${ }^{7}$.

De esta manera la familia, como forma societaria básica y neta, da paso a una forma societaria donde se regula que algunos de los participantes en la reproducción han de quedar -o pueden quedar- en el anonimato, desaparecen y de ellos no se conoce -salvo en casos estrictamente necesarios- su identidad, pues su intervención se limita a la finalidad de ayudar en el proceso reproductivo, pero no juega un papel en relación con el niño fruto de la donación. Sin embargo, pese al anonimato, su presencia "planea" sobre el grupo familiar, pues se entabla una relación que ya no se puede abandonar, recogiendo algunas normativas la posibilidad de facilitar la información en coyunturas excepcionales (cuando, por ejemplo, medie la posible curación de una enfermedad genética) ${ }^{8}$.

Además, con la reproducción asistida conceptos sociales cercanos e íntimos para los seres humanos como la maternidad, la paternidad, las estructuras del parentesco y la propia concepción tradicional de la familia se han trastocado.

\section{Reprogenética y Demografía}

La Reprogenética ha comprometido a la Demografía, en concreto a la Demografía dinámica ${ }^{9}$ y a algunos de sus principales indicadores. Ya se planteó hace algunos años que las técnicas de reproducción asistida podrian ser una solución viable para mitigar los efectos del envejecimiento de la población europea ${ }^{10}$, teniendo en cuenta que sería factible planificar técnicamente la natalidad.

\subsection{La tasa de fertilidad}

La noción de la tasa de fertilidad definida como -el número de hijos que las mujeres pueden tener en términos biológicos- se ha visto concernida, puesto que ya no existen límites para que mujeres menopáusicas y/o mayores gesten y alumbren hijos ${ }^{11}$. Ateniéndonos a los datos relativos a las proyecciones demográficas sobre las tasas de fertilidad en los países desarrollados para los próximos años $^{12}$, no puede inferirse -de momento-que las técnicas de reproducción asistida vayan a tener una incidencia cuantitativa sobre el número de nacimientos pero, en algunos casos concretos, el concepto de fertilidad en su formulación clásica está comprometido, puesto que ya no hay limitaciones para que mujeres mayores o menopáusicas tengan descendencia.

Especial repercusión pública tuvo, por ser uno de los primeros casos conocidos, el nacimiento en el año 1994, en 
Italia, de un niño de una mujer de sesenta y tres años, o el de una mujer hindú que con 65 años trajo al mundo en 2003 a un bebé gracias a la reproducción asistida. En España alcanzó una especial repercusión mediática el caso de María del Carmen Bousada del Lara de 67 años, que en 2006 dio a luz a gemelos en un hospital barcelonés, tras someterse a un tratamiento de fertilidad en la clínica Pacific Fertility Center de Los Ángeles. En 2009 se hizo público su fallecimiento por cáncer, reabriéndose el debate médico, ético, moral y social sobre la maternidad a edad avanzada.

Las clínicas de reproducción asistida admiten a mujeres hasta una edad en torno a los cincuenta años, coincidiendo con el período fértil femenino. Hay reservas médicas a que mujeres que han sobrepasado esta edad sean madres, debido a los riesgos para el embrión/bebé en formación (posible hipotrofia del feto, diabetes tipo I, etc.), o a los problemas médicos derivados del embarazo para la madre (fundamentalmente, alta presión arterial). También deben consignarse los inconvenientes psicológicos que puedan surgir entre las partes implicadas, como consecuencia de la excesiva diferencia generacional o la posibilidad, hecha realidad en el caso de M. a de Carmen Bousada, de dejar pronto huérfanos a los pequeños.

La Iglesia Católica ha mostrado su disconformidad con la utilización de estas técnicas en estos casos, pues estima que es contraria a la dignidad humana, porque la persona (nasciturus) debe ser siempre un sujeto y fin en sí mismo.

\subsection{La tasa de fecundidad}

Otro concepto tan relevante como es el de la fecundidad - que es el número de hijos nacidos vivos que tiene por término medio una mujer- se está viendo también involucrado, en la medida que los diagnósticos preimplantatorios ${ }^{13}$ y los diagnósticos prenatales ${ }^{14}$, recomendados en nuestro país a las mujeres a partir de los treinta y cinco años, introducen una dimensión de "control de calidad", que no debemos menospreciar.

Por otro lado, el diagnóstico genético preimplantacional ha abierto nuevas vías en la prevención de determinadas enfermedades genéticas. En algunos casos, con el control y la autorización administrativa pertinente, están siendo utilizados para intentar salvar la vida de familiares enfermos ${ }^{15}$.

En octubre de 2008 -con una extraordinaria repercusión pública- nació el hasta ahora único niño concebido en España tras un diagnóstico preimplantacional. Se buscaba la histocompatibilidad con su hermano enfermo (DGP+HLA). Desde el año 2006 hasta la actualidad la Comisión Nacional de Reproducción Asistida ${ }^{16}$, ha dado informes positivos a más de una docena de peticiones de DGP+HLA (fundamentalmente de pacientes afectados de enfermedades como la betatalasemía mayor, la anemia de fanconi, etc.), en función del debido respeto a los criterios éticos y científicos marcados por la ley. Especialmente, el DGP+HLA, según el Apartado 2 del Artículo 12 de la Ley 14/2006, requiere la autorización expresa, "caso a caso", teniendo en cuenta "las caracteristicas clínicas, terapéuticas y sociales de cada uno de ellos".

En consecuencia, en países como España es factible que nazcan más niños vivos y sanos por año, ya que es posible seleccionar los preembriones ${ }^{17}$ en fases muy incipientes de desarrollo $y$, en su caso, recurrir al aborto terapéutico cuando el feto está afectado por alguna enfermedad genética o cromosopatía de gravedad.

Todo lo anterior, junto a los avances médicos en general, está afectando a las tasas de mortalidad infantil, con una reducción significativa de las mismas en los países más avanzados. Asimismo, es notable la disminución del nacimiento de personas con graves discapacidades de origen genético, como consecuencia entre otros factores de los avances en materia preventiva ${ }^{18}$.

Uno de los temas más controvertidos en genética clínica es la posibilidad de seleccionar el sexo en la descendencia. Su práctica generalizada podria mudar la estructura del grupo familiar y los actuales equilibrios demográficos, de acuerdo a las preferencias de los progenitores o de grupos particulares que estarian en disposición, por ejemplo, de elegir tener hijos varones de manera descompensada ${ }^{19}$. Por estas razones, existe un consenso internacional (al menos entre los países más desarrollados) para que este procedimiento sólo sea utilizado bajo estrictas razones terapéuticas. En ese sentido se manifiesta la Ley Española de Reproducción Asistida, en donde se tipifica como infracción muy grave "la selección del sexo o la manipulación genética 
con fines no terapéuticos o terapéuticos no autorizados" (artículo 26.C.10.a).

El progresivo y cada vez más completo conocimiento de nuestras bases genéticas y el perfeccionamiento de los diagnósticos y terapias consecuentes también incidirán en la esperanza media de vida. La utilización de células madre, especialmente de células troncales embrionarias (potencialmente con capacidad para generar cualquier tipo de tejido), ha abierto nuevas expectativas en la curación -o mejora- de patologías hoy en día incurables (Parkinson, Alzheimer, diabetes, osteoporosis, paraplejias, insuficiencias cardiacas, etc.). Por otro lado, la clonación no reproductiva ${ }^{20}$ ofrece también posibilidades curativas, resultando una técnica que ha suscitado un gran debate ético.

De forma que, si a los diagnósticos preimplantacionales y prenatales añadimos las posibilidades de desarrollo y potenciación a medio plazo de una medicina predictiva, el hombre del futuro ganará en calidad de vida y, llegado el caso, la esperanza media de vida podría alcanzar cotas actualmente impensables (consecuencia de la reprogramación de la muerte celular). Lo cual se acabaría traduciendo no sólo en un aumento estático de la población mundial, sino también en nuevos patrones de distribución y en un progresivo envejecimiento de las pirámides de población.

En definitiva, la expansión del uso de la Reprogenética podría acabar modificando las pirámides de la población mundial, conduciendo a un incremento sustancial de las cohortes de personas de mayor edad; produciendo un desequilibrio entre varones y mujeres y contribuyendo a la disminución del número de personas afectadas por patologías de origen genético. Todo lo cual plantea cuestiones relacionadas con la necesidad de redefinir conceptos demográficos básicos, así como evaluar los efectos que se derivarían de la eventual planificación de los procesos demográficos en función de criterios e intereses particulares.

Las tendencias previsibles trascienden, como vemos, el plano meramente demográfico y nos sitúan ante interrogantes como: ¿qué riesgos demográficos introducen?, ¿dónde establecer los límites entre lo patológico y lo sano?, ¿se están introduciendo criterios de "control de calidad" en los seres humanos cuando, por ejemplo, se recurre a los diagnósticos preimplantacionales y prenatales?, ¿hacia qué sociedades nos pueden conducir estas técnicas ${ }^{21}$ ?, etc.

\section{Reprogenética y familia}

En un primer nivel de análisis hay que indicar que las técnicas de reproducción asistida alteran la especificidad tradicional de la familia de constituir una "comunidad de sangre". Como ya hemos subrayado, en nuestros días, los límites biológicos del grupo familiar se pueden traspasar. Basta para ello con que se utilice material genético de donantes, con lo que se da entrada a otros participantes anónimos que no llegarán a ser miembros del núcleo familiar, pero tienen un gran protagonismo en estos nuevos procesos reproductivos ${ }^{22}$.

La vinculación biológica, que ha tenido tanto peso en nuestra cultura, y ha sido uno de los rasgos definitorios del grupo familiar, con estas técnicas ve reducido su papel en favor de la vinculación socio-cultural, de los lazos de afecto y de los intereses comunes derivados de la relación psicológica que se deriva del hábito de vivir juntos.

Tönnies vio con claridad este papel cuando subrayó que "... la casa constituye la sede y el cuerpo del parentesco... La voluntad y el espíritu de parentesco no quedan confinadas a los muros de la casa, ni ceñidos a la proximidad física, les repugna la separación, ya que la sola proximidad satisface plenamente el deseo afectivo" (Tönnies, 1979: 39-40). También Durkheim, haciendo alusión a los clanes, sostuvo que sus integrantes forman entre sí una familia, a pesar de que "... para formar parte de ella no es necesario tener con los otros miembros del clan relaciones de consanguineidad" (Durkheim, 1973: 153). Planteamientos que contrastan con la concepción de George Simmel cuando sostiene que "... en la moderna vida de familia, aflojada en su lazos, atomizada...., lo único que todavía caracteriza a la familia es la comunidad fisiológica y la herencia biológica estrechamente unida a ésta..." (Simmel, 1977: 529).

Este tema adquiere un nivel de complejidad mayor en el caso de los preembriones crioconservados, tratándose de una de las posibilidades que mayor controversia ha tenido a lo largo de estas décadas de praxis de la reproducción asistida. Según el artículo 11.3 de la Ley 35/1988 so- 
bre Técnicas de Reproducción Asistida, "los preembriones sobrantes de una FIV, por no transferidos al útero, se crioconservarán en los Bancos autorizados, por un máximo de cinco años". Casi dos décadas después en España se resuelve esta problemática, al contemplarse en la Ley 14/2006 diversas posibilidades para estos preembriones: bien utilizarlos por la propia mujer o su cónyuge, donarlos con fines reproductivos, donarlos con fines de investigación o el cese de su conservación sin otra utilización, siempre y cuando se haya finalizado el plazo máximo de conservación establecido en la Ley sin que se haya optado por alguna de las opciones previas. Resulta reseñable que por Ley son los responsables médicos, con el dictamen favorable de especialistas independientes y ajenos al Centro correspondiente, los que deciden cuando dar por finalizada su crioconservación.

Debe tenerse en cuenta que estas técnicas conllevan que conceptos sociales primarios cercanos e íntimos para los seres humanos como son la maternidad, la paternidad, las estructuras del parentesco y la propia concepción de familia se están modificando. Así las cosas, aparecen nuevas figuras de madres/padres, que alcanzan ese estatus a partir de una intermediación científico-tecnológica, que busca, por encima de todo, el resultado. Un resultado que debe materializarse con la llegada al mundo de un nuevo individuo, del que por ley, el equipo médico deberá procurar garantizar la mayor similitud fenotípica e inmunológica con la mujer receptora ${ }^{23}$.

Hasta la extensión de los métodos anticonceptivos y la aparición de estas técnicas, los hijos eran recibidos como "un don de Dios" y en las familias venían al mundo "cuantos hijos Dios quería". En la actualidad, al menos en los países más desarrollados, los hijos son el resultado voluntario del deseo de maternidad y paternidad de parejas e individuos concretos.

\subsection{Principales efectos de las técnicas de reproducción asistida sobre la familia}

Para entender adecuadamente algunos de los principales efectos de las modernas técnicas reproductivas sobre la familia, resulta pertinente considerar la variada casuística sociológica que ha ido apareciendo desde que en 1978 nació la primera niña probeta del mundo, Louise July Brown.
2.1.1. La inseminación artificial, la fecundación asistida $y$ técnicas afines en parejas heterosexuales con y sin utilización de donantes

El caso que presenta menos complejidad, al tiempo que menor conflictividad desde el punto de vista social, ético, jurídico y personal es el de la inseminación artificial, fecundación "in vitro" y técnicas afines, empleadas en el ámbito de parejas heterosexuales casadas/parejas de hecho, que utilizan su propio material genético ${ }^{24}$. El grupo familiar es la "unidad reproductora", los derechos y deberes de sus miembros están garantizados por ley de la misma manera que en una fecundación "in vivo" y al hijo/a no le falta el referente materno y paterno. El esquema familiar se ajusta al de una familia tradicional: madre biológica-legal-social (esposa, misma persona); padre biológico-legal-social (esposo, misma persona); se da una interconexión entre los miembros, con parientes maternos y paternos y el nacido, considerado hijo natural, disfruta de todos los derechos/ deberes derivados de su condición. En situación similar estarian las parejas de hecho legalizadas que acudieran a estas técnicas para tener descendencia, con un esquema familiar idéntico (vid. Cuadro 2).

En los dos casos anteriores la situación adquiriria mayor complejidad si se precisara la intervención de donantes externos al núcleo familiar, resultando modificado el esquema familiar, en comparación con el anterior, así tendríamos a: la madre biológica (donante, persona diferente)-legal-social (esposa, misma persona); el padre biológico (donante, diferente persona)-legal-social (misma persona). Se da una interconexión entre los miembros familiares derivada de la filiación legal, con parientes maternos (en ningún caso, de acuerdo a la ley, con los de la donante de óvulos, que es la madre biológica) y paternos (en ningún caso, de acuerdo a la ley, con los del donante de semen, que es el padre biológico), y el nacido disfruta de todos los derechos/deberes derivados de su condición (vid. Cuadro 3).

\subsubsection{La inseminación artificial, la fecundación asistida y técnicas afines en mujeres solas y parejas homosexuales femeninas}

Dos posibilidades que presentan un cierto nivel de dificultad desde el punto de vista social, ético, jurídico y personal se concretan en la posible utilización de estas técnicas por 
CUADRO 2: EFECTOS DE LA INSEMINACIÓN ARTIFICIAL, LA FECUNDACIÓN IN VITRO Y TÉCNICAS AFINES SOBRE LA FAMILIA EN EL CASO DE PAREJA HETEROSEXUAL CASADA/PAREJA LEGALIZADA QUE EMPLEA SU PROPIO MATERIAL GENÉTICO

\begin{tabular}{|l|}
\hline - El grupo familiar es la "unidad reproductora". \\
\hline - Los derechos y deberes de sus miembros garantizados por ley. \\
\hline - El esquema familiar se ajustaría al de una familia tradicional: \\
- Madre biológica-legal-social (esposa/mujer de la pareja, misma persona) \\
- Padre biológico-legal-social (esposo/varón de la pareja, misma persona) \\
- Interconexión entre los miembros de la familia. \\
- Con parientes maternos y paternos. \\
- El nacido disfrutaría de todos los derechos/deberes derivados de su condición. \\
\hline
\end{tabular}

Fuente: Elaboración propia.

\section{CUADRo 3: EFECTOS DE LA INSEMINACIÓN ARTIFICIAL, LA FECUNDACIÓN IN VITRO Y TÉCNICAS AFINES SOBRE LA FAMILIA EN EL CASO DE PAREJA HETEROSEXUAL CASADA/PAREJA LEGALIZADA QUE EMPLEA MATERIAL GENÉTICO DE DONANTES}

\begin{tabular}{|l|}
\hline - La unidad familiar no es la "unidad reproductora". \\
\hline - Los derechos y deberes de sus miembros garantizados por ley. \\
\hline - El esquema familiar se ajustaría al de: \\
- Madre biológica (donante de óvulos, persona diferente)-legal-social (esposa/mujer de la pareja, misma persona) \\
- Padre biológico (donante de semen, persona diferente)-legal-social (esposo/varón de la pareja, misma persona) \\
- Interconexión entre los miembros de la familia derivada de la filiación legal-social. \\
- Con parientes de la madre y el padre legal-social. \\
-El niño disfrutaria de derechos/deberes derivados de su condición \\
\hline
\end{tabular}

Fuente: Elaboración propia.

mujeres solas (viudas, solteras, etc.) o por parejas homosexuales de mujeres. En el primer caso, el esquema familiar resultante sería el de una madre biológica-jurídica-social (la misma persona), un padre biológico desconocido (donante), sin padre social, sin parentela paterna y sólo con parentela materna (vid. Cuadro 4).

CUADRo 4: EFECTOS DE LA INSEMINACIÓN ARTIFICIAL, LA FECUNDACIÓN IN VITRO Y TÉCNICAS AFINES SOBRE LA FAMILIA EN EL CASO DE PAREJA HETEROSEXUAL CASADA/PAREJA LEGALIZADA QUE EMPLEA MATERIAL GENÉTICO DE DONANTES

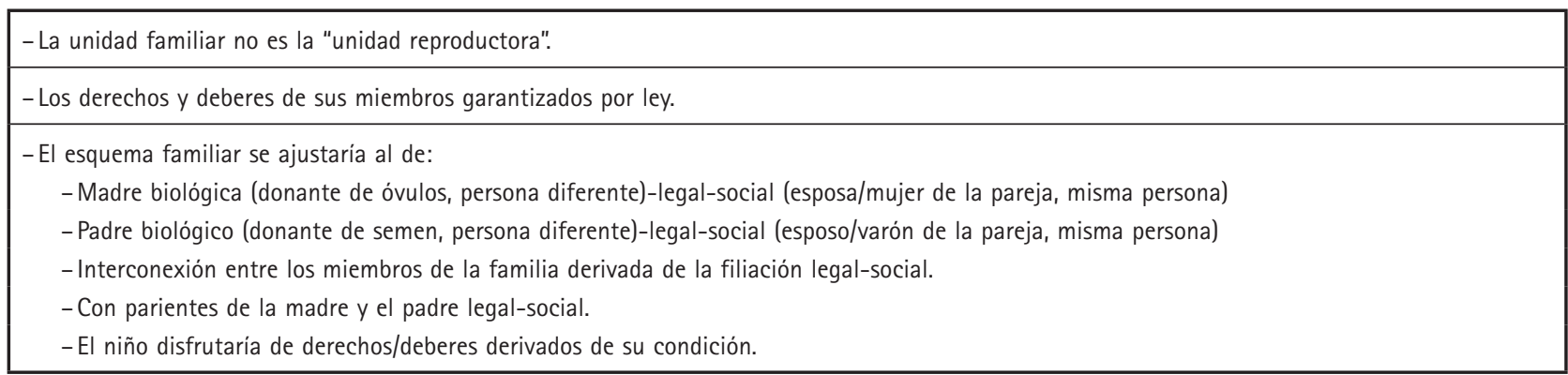

Fuente: Elaboración propia. 
En el caso de su utilización por parejas homosexuales de mujeres (casadas y parejas de hecho), que utilicen su propio material genético, recurriendo a bancos de semen, el esquema familiar es: una madre biológica-legal-social (misma persona, la gestante ${ }^{25}$ ); un padre biológico-legal- social (no existiría, donante); se da una interconexión entre los miembros, con parientes de las dos figuras familiares (cónyuge a y cónyuge b) y el nacido disfruta de todos los derechos/deberes derivados de su condición ${ }^{26}$ (vid. Cuadro 5).

\section{CUADRo 5: EFECTOS DE LA INSEMINACIÓN ARTIFICIAL, LA FECUNDACIÓN IN VITRO Y TÉCNICAS AFINES SOBRE LA FAMILIA EN EL CASO DE PAREJA HOMOSEXUAL FEMENINA CASADA/PAREJA LEGALIZADA QUE EMPLEA SEMEN DE DONANTE}

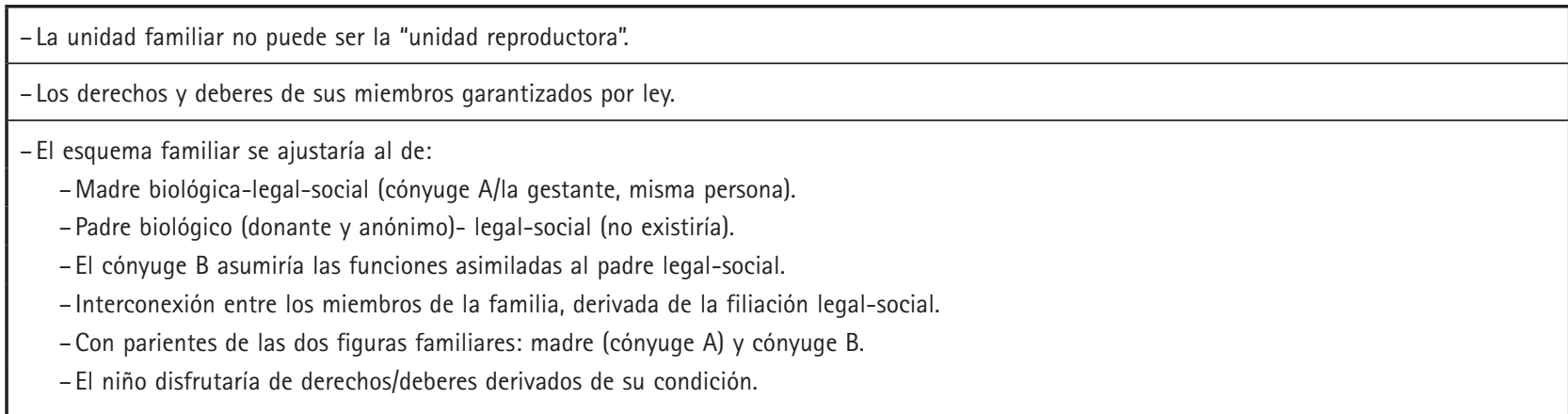

Fuente: Elaboración propia.

En el caso anterior cabe introducir otra variable adicional, que la pareja homosexual femenina casada o pareja de hecho, utilice semen y óvulos de donantes. El esquema familiar y la relación de parentesco se ajusta al de: una madre biológica (desconocida/donante)-legal (la que alumbra dentro de la pareja)-social (la pareja de mujeres); un padre biológico (desconocido y sería el donante de semen)-legal-social (no existiría); con parientes de las dos integrantes de la pareja femenina y sin parientes derivados de la figura masculina paterna. El nacido disfruta de todos los derechos/deberes derivados de su condición (vid. Cuadro 6).

CUADRO 6: EFECTOS DE LA INSEMINACIÓN ARTIFICIAL, LA FECUNDACIÓN IN VITRO Y TÉCNICAS AFINES SOBRE LA FAMILIA EN EL CASO DE PAREJA HOMOSEXUAL FEMENINA CASADA/PAREJA LEGALIZADA QUE EMPLEA SEMEN Y ÓVULOS DE DONANTE

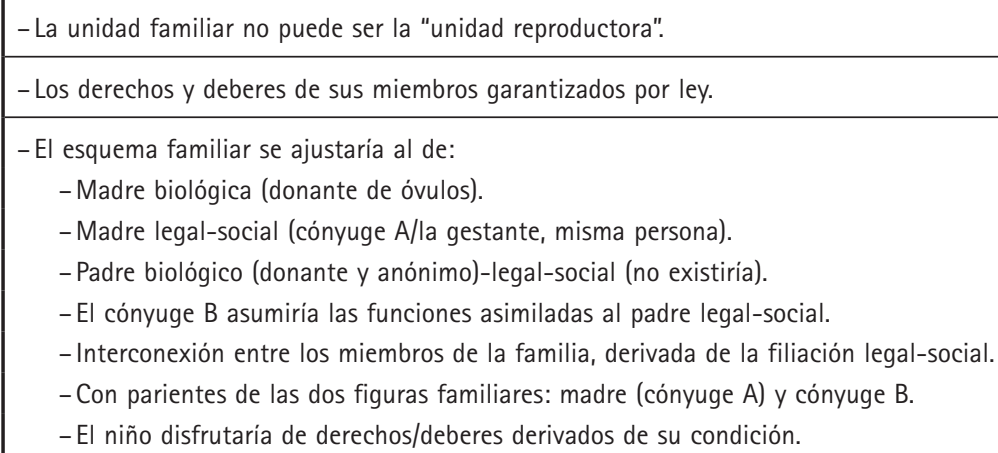

Fuente: Elaboración propia. 
Un caso que saltó hace varios años a la opinión pública española fue el de una pareja de lesbianas que solicitó autorización para una fecundación asistida, utilizando los óvulos de una de las integrantes de la pareja acudiendo a semen de donante, con la particularidad que solicitaban que los óvulos fecundados no fueran transferidos a la madre biológica, sino a la otra mujer. De esa forma, querían asegurar que ambas participarian en el proceso reproductivo. La Comisión Nacional de Reproducción Asistida emitió informe negativo, por entender que era un caso asimilado a la maternidad subrogada, que la donación siempre es anónima y, por tanto, no podía ser autorizado por ley. De haberse permitido $o$ en aquellos paises donde no haya impedimentos legales el esquema familiar y la relación de parentesco se ajusta al de: una madre biológica (la que proporciona el óvulo), una madre legal (la que alumbra dentro de la pareja), dos madres sociales (ambas mujeres); un padre biológico (desconocido y es el donante de semen), un padre legal (no existe), un padre social (no existe); con parientes de las dos integrantes de la pareja de mujeres y sin parientes derivados de la figura masculina paterna. El nacido disfruta de todos los derechos/ deberes derivados de su condición (vid. Cuadro 7).

CUADRO 7: EFECTOS DE LA INSEMINACIÓN ARTIFICIAL, LA FECUNDACIÓN IN VITRO Y TÉCNICAS AFINES SOBRE LA FAMILIA EN EL CASO DE PAREJA HOMOSEXUAL FEMENINA CASADA/PAREJA LEGALIZADA QUE UTILIZA SEMEN DE DONANTE, ÓVULOS DE UNA DE ELLAS Y EL EMBRIÓN SE TRANSFIERE A LA OTRA

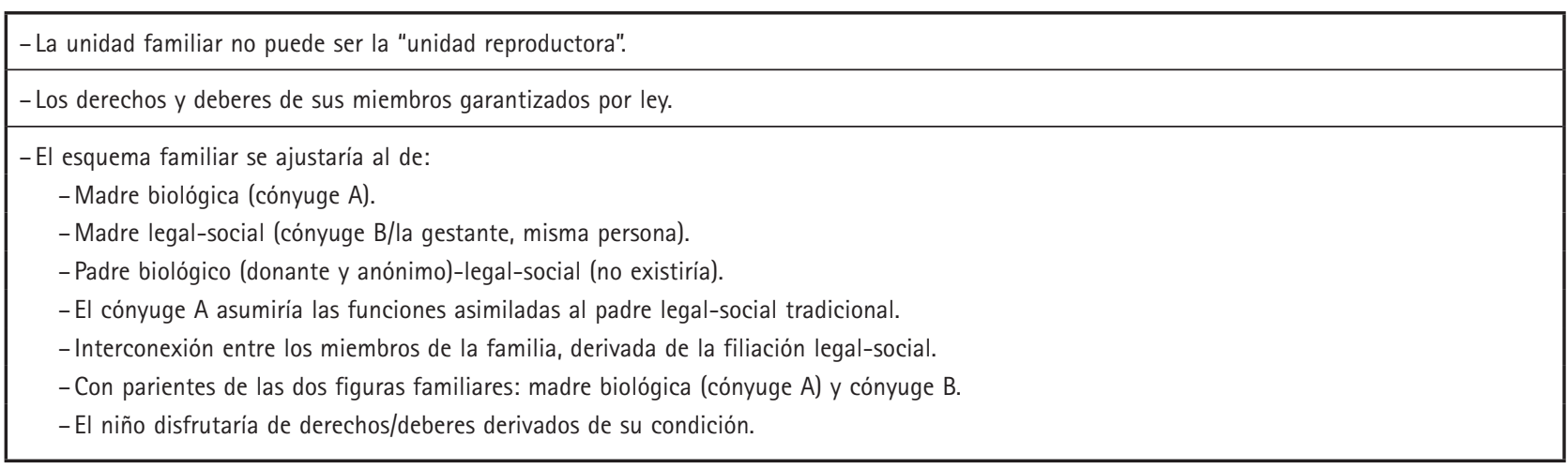

Fuente: Elaboración propia.

\subsubsection{La fecundación asistida y técnicas afines en transexuales casados}

Otro caso que despertó gran interés en la opinión pública internacional el año 2009 fue el caso de Thomas Beata, un transexual de 34 años, nacido mujer, que transformó su aspecto físico e identidad jurídica en un varón, pero decidió mantener sus órganos genitales y reproductivos femeninos. En el año 2008 dio a luz a su hija Susana, concebida a través de un procedimiento de inseminación artificial. Además, el (la) joven estaba casado/a con una mujer imposibilitada para tener descendencia. El esquema familiar y la relación de parentesco se ajustó al de: una madre biológica (transexual)-legal (la que alumbra dentro de la pareja, transexual)-social (la mujer transexual y su esposa); un padre biológico (desconocido y es el donante de semen)legal-social (no existe); con parientes de las dos integrantes de la pareja y sin parientes derivados de la figura masculina paterna. La nacida disfruta de todos los derechos/deberes derivados de su condición (vid. Cuadro 8).

\subsubsection{La maternidad subrogada}

Un tema destacable, desde el punto de vista sociológico, es el de la gestación por sustitución. Se trata de una de las derivaciones que mayores controversias suscita. En ella la madre de "alquiler" presta su vientre a un matrimonio/pareja legal heterosexual/homosexual o a una mujer u hombre solo, y sus servicios culminan con el nacimiento de un bebé.

El caso más sencillo es el de una pareja heterosexual casada o legalizada que desea tener descendencia. A la participación dentro de la unidad familiar de un individuo ajeno al grupo familiar (la madre de alquiler) se puede 

DE PAREJA CASADA INTEGRADA POR UN TRANSEXUAL Y UNA MUJER

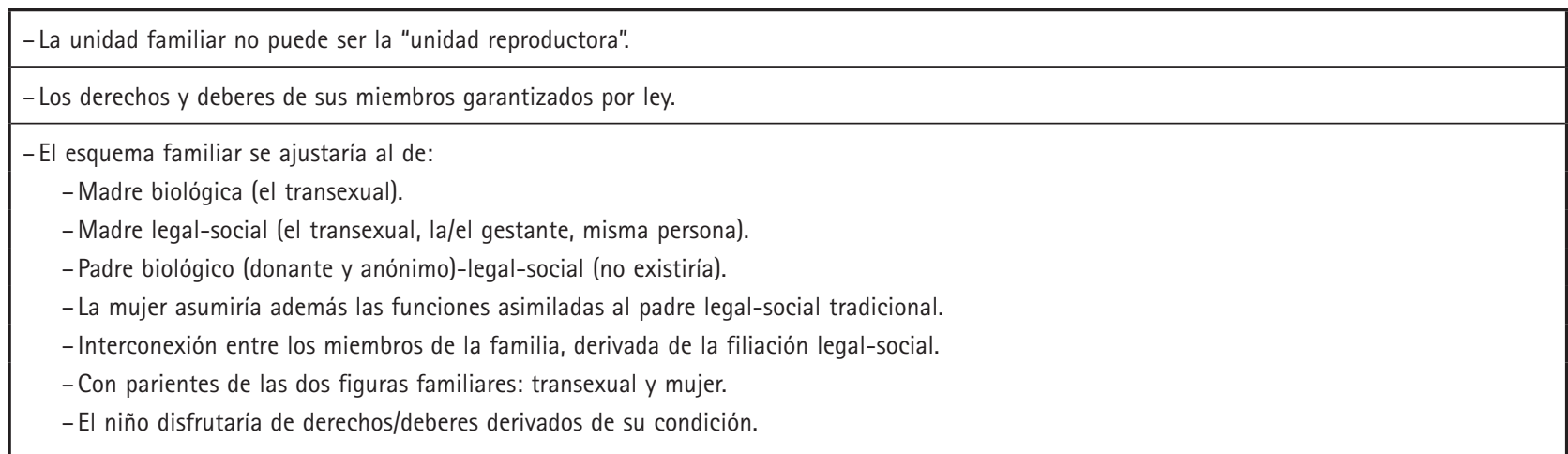

Fuente: Elaboración propia.

añadir la intervención de otros individuos, complejizándose considerablemente el entramado de parentesco. El cruce de grupos adquiere una gran significación, pudiendo aparecer tres padres: el padre biológico (el que aporta el semen, bien del varón que contrata junto a su mujer los servicios de la madre subrogada, bien del marido/pareja legal de la madre subrogada o de un donante anónimo), el padre legal (el marido/pareja legal de la mujer que subroga su útero) y el padre social (el marido/pareja legal de la pareja que, junto con su mujer/pareja legal, contrata los servicios de la madre subrogada). Pero además podrian convivir tres madres, en sus diversas variantes, la madre biológica (de la que fuera el material genético, bien la madre subrogada, una donante anónima o la mujer/pa- reja legal que contrata junto a su marido/pareja legal los servicios de esta mujer), la madre jurídica (por ley siempre la que alumbra y sería la madre subrogada) y la madre social (la que contrata junto a su marido/pareja legal los servicios de la mujer subrogada). El nacido tiene parentela materna y paterna, derivada del desempeño de la maternidad y paternidad sociales y el niño disfruta de todos los derechos/deberes derivados de su condición, siempre y cuando se respete el contrato de subrogación. Desde el punto de vista familiar/parental la situación adquiere otros matices y dimensiones en el caso de que fueran parejas homosexuales casadas o legalizadas y mujeres $u$ hombres solos los que utilizaran los servicios de mujeres que subrogaran sus úteros (vid. Cuadro 9).

CUADRo 9: EFECTOS DE LA INSEMINACIÓN ARTIFICIAL, LA FECUNDACIÓN IN VITRO Y TÉCNICAS AFINES SOBRE LA FAMILIA EN EL CASO DE PAREJA HETEROSEXUAL CASADA/PAREJA LEGALIZADA QUE RECURRE A LOS SERVICIOS DE UNA MADRE SUBROGADA

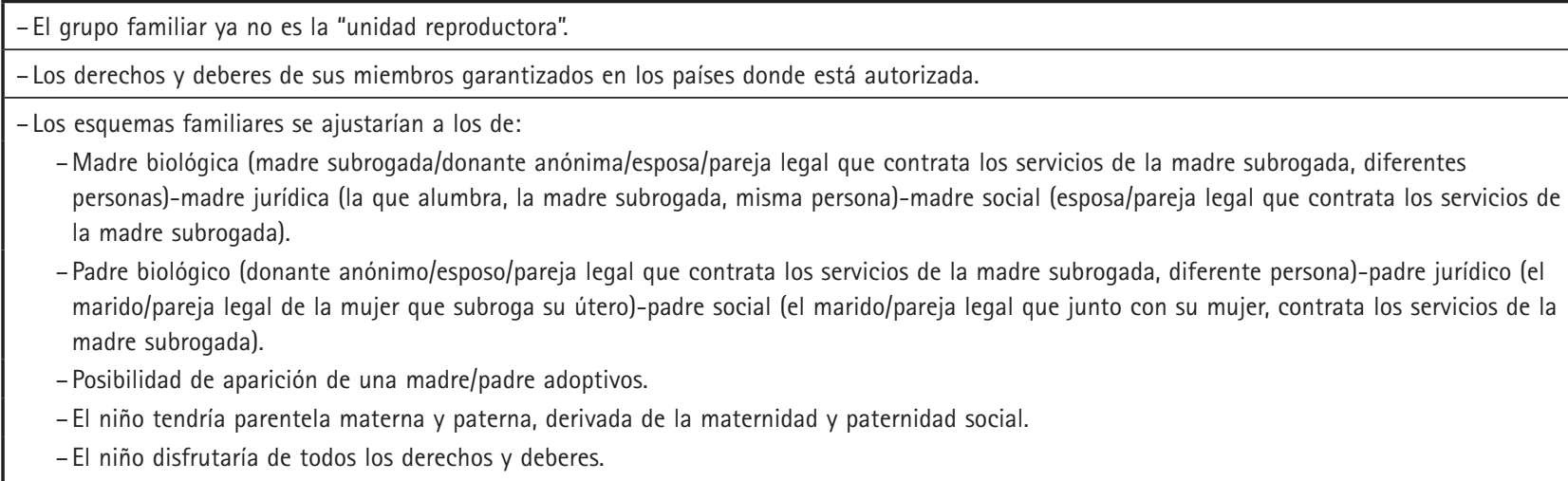

Fuente: Elaboración propia. 
Una variedad de la subrogación de úteros se concreta en las posibilidades de que alguna de las madres de uno de los integrantes de una pareja heterosexual/homosexual o de una mujer sola o varón solos, en definitiva, las abuelas gesten y alumbren a sus propios hijos-nietos. Los primeros casos conocidos fueron el de Arteta Schweitzer, una norteamericana de 42 años que en el año 1991 dio a luz a sus propios nietos, hijos biológicos de su hija y su yerno; y en 1992 el de Geraldine Mesolowsky, una mujer menopáusica de 53 años, que paría a su nieto, hijo también genético de su yerno y de su hija. En ambos casos se integra dentro de la unidad familiar conyugal un individuo integrante del propio grupo familiar (abuela-útero subrogado), complejizándose el entramado de parentesco. El grupo familiar es la unidad reproductora, los derechos y deberes de sus miembros están garantizados en los paises donde está autorizada. El esquema familiar, salvo que se incorporaran donantes de material genético en sus diversas variantes resultaría del siguiente modo: la madre biológica sería la esposa/pareja legal del varón que utiliza los servicios de alguna de sus madres (abuelas); la madre legal sería la madre subrogada (abuela), la madre social la esposal pareja legal del varón que utiliza los servicios de alguna de sus madres (abuelas), un padre biológico el esposo/ pareja legal (yerno) de la mujer que utiliza los servicios de alguna de sus madres (abuelas), un padre jurídico que sería el esposo de la abuela (abuelo) y un padre social que sería el yerno. El niño tendría parentela materna y paterna, derivada de la maternidad y paternidad biológica, juridica y social y disfrutaría de todos los derechos derivados de su condición. Como vemos, el cruce de grupos cobra especial significación, se desdibujan las líneas materna y paterna y se produce un confusionismo derivado de la maternidad y paternidad jurídicas (vid. Cuadro 10).

De lo que no cabe duda es que la gestación por sustitución involucra la lógica tradicional de la familia e impacta sobre las estructuras del parentesco, quebrando -y en algunos casos, complejizando en exceso- la conceptualización heredada de familia en sentido tradicional.

\section{CUADRO 10: EFECTOS DE LA INSEMINACIÓN ARTIFICIAL, LA FECUNDACIÓN IN VITRO Y TÉCNICAS AFINES SOBRE LA FAMILIA EN EL CASO DE PAREJA HETEROSEXUAL CASADA/LEGALIZADA QUE RECURRE A LOS SERVICIOS DE UNA MADRE SUBROGADA OUE ES LA ABUELA DEL NACIDO UTILIZANDO SU PROPIO MATERIAL BIOLÓGICO}

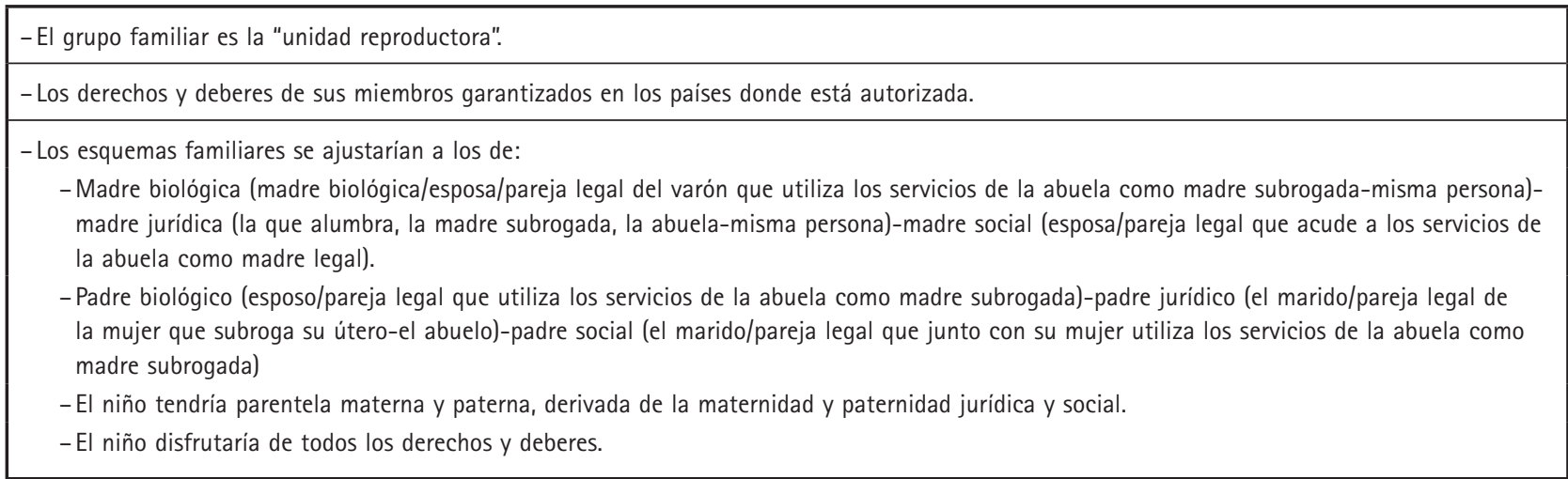

Fuente: Elaboración propia.

\subsubsection{La clonación reproductiva}

Una técnica, por el momento no autorizada por ninguna de las legislaciones del mundo ${ }^{27}$, es la clonación reproductiva. Se trata de un procedimiento cuyo objetivo es generar in- dividuos clónicos, que está siendo reivindicado por algunos especialistas en reproducción asistida ${ }^{28}$, pues consideran que el así nacido sería genéticamente descendiente del sujeto al que se le realizara esta técnica, no precisándose la intervención de donantes de gametos. 
La utilización de la clonación reproductiva en humanos produce inquietud, por las reservas éticas y morales asociadas a la generación "en serie" de personas supuestamente iguales. El niño/a nacido tras un procedimiento de clonación genotípicamente sería idéntico al individuo del cual posee su carga genética, pero en realidad se trataría de otra persona. Se sentiría como un individuo diseñado ex profeso por terceras personas, y su situación no sería en ningún caso equivalente a los casos de gemelos idénticos ${ }^{29}$.

Con las eventuales clonaciones, la familia quedaría también seriamente afectada, al desdibujarse las líneas parentales y genealógicas. Se eliminaría del proceso reproductivo a uno de los sexos, la reproducción en la especie humana sería el resultado de relaciones asexuadas, fruto de un proceso técnico-cultural, en donde la maternidad y paternidad biológicas dejarían de ser el referente de la transmisión de la vida, en un entorno en donde los seres humanos potencialmente estaríamos en disposición de generar vida sin límites.
La utilización de la clonación, sin limitaciones de edad, por parte de parejas heterosexuales/homosexuales y mujeres y hombres solos, asimismo daría lugar a una casuistica variada y compleja, con efectos evidentes sobre la familia. Centrémonos en uno de los casos de menor nivel de complejidad, el de un matrimonio/pareja legal que, tras haber fracasado con los tratamientos en reproducción asistida, decidiera recurrir a la clonación reproductiva.

El esquema familiar y parental sería el siguiente: una madre biológica (de la que se utilizan las células para el procedimiento de clonación)-legal (la que alumbra)-social (misma madre); un padre biológico (no habría)-legal (el marido/pareja legal de la mujer de la que se utilizan las células para el procedimiento de clonación)-social (el marido/pareja). También se podría dar el caso de que la clonación se realizara a partir del material biológico del varón. En un caso así, no habría un padre biológico en sentido tradicional, si bien sí legal y social. El nacido tendría parentela por vía materna y paterna (vid. Cuadro 11)

\section{CUADRo 11: EFECTOS DE LA CLONACIÓN SOBRE LA FAMILIA EN EL CASO DE PAREJA HETEROSEXUAL CASADA/PAREJA LEGALIZADA QUE UTILIZARA PARA EL PROCEDIMIENTO CELULAS DEL VARÓN}

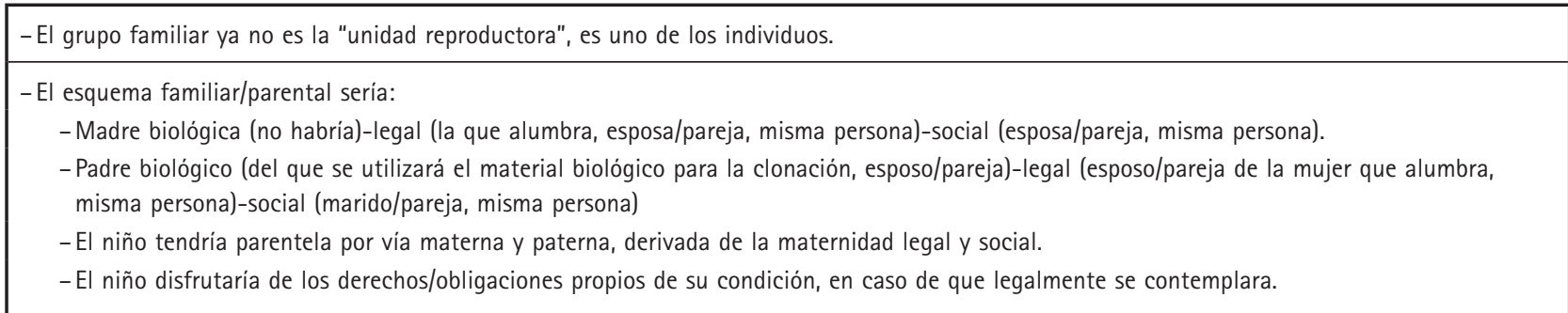

Fuente: Elaboración propia.

Por el momento, la clonación se sitúa más en el plano de las posibilidades que en el de la realidad más inmediata, y habrá que ver cómo discurren los avances científicos y cuáles son los parámetros sociales y éticos vertebradores de las sociedades a medio y largo plazo.

A la luz de lo expuesto, a diferencia de la reproducción tradicional, en donde la conceptualización y figura del padre/madre, además de la filiación y las relaciones de parentesco están determinadas con claridad, es obvio que las técnicas de reproducción asistida dan lugar -y darán- $a$ incertidumbres y cambios sociales profundos ante la imposibilidad de ubicar correctamente desde nuestros parámetros culturales las nuevas posibilidades abiertas a la maternidad, la paternidad, a la definición de la filiación y a la consideración de las nuevas relaciones de parentesco surgidas (Heritier, 1998).

Hace ya veinticinco años el profesor Botella Llusía advirtió que: 
"... con la tecnología de la reproducción, la especie humana puede cambiar su estructura y sus relaciones, desapareciendo la unión de hombre y mujer como pareja básica, como unión básica en la vida, sea religiosa o no lo sea, de suerte que se pierda el sentido que el sexo masculino y femenino tengan en la sociedad del futuro" (Comisión Especial de Estudio sobre la Fertilización Extracorpórea, 1985).

\section{Algunas conclusiones}

Todo parece indicar que la Reprogenética irá unida a nuevos planteamientos y concepciones sobre el ser humano, la familia, las relaciones sociales y de parentesco, las ciencias y las sociedades. No es suficiente con subrayar la originalidad de las investigaciones particulares. Es necesario mostrar, en toda su amplitud, que de igual modo que el siglo XX fue el siglo de la física, el siglo XXI puede ser el de la biotecnología, en general y el de la Reprogenética, en particular. Lo cual exigirá la formalización de una estructura conceptual e intelectual que se corresponda con los rasgos de la nueva sociedad emergente. Esa nueva sociedad se verá precisada a modificar la naturaleza de la propia organización social, y los sociólogos tendrán que responder a interrogantes del tipo ¿cómo será esta sociedad en ciernes donde convergen lo biológico-genético con lo social?, ¿cuál será su naturaleza?, ¿cuáles serán las instituciones de una sociedad de esas características y cuáles sus fuerzas dominantes?, ¿cómo afectará y modificará a la familia?, ¿en qué medida se verán alterados los principales indicadores demográficos?, ¿cuáles serán las cualidades particulares de las relaciones sociales?

Las posibilidades abiertas con la Reprogenética tienen que contextualizarse dentro de un proceso social asociado a la secularización de la cultura. Dentro de este proceso está surgiendo una nueva concepción y valoración de la Naturaleza, particularmente biológica, sujeta a la intervención humana, con el riesgo de sobrepasar su dominio y poner en riesgo la especie humana, tal como hoy la conocemos. También la Reprogenética conlleva una modificación de la actual concepción de la Medicina y de sus alternativas, con posibles efectos discriminatorios basados en la herencia genética. De ahí la importancia de regular normativamente y establecer mecanismos de seguimiento y control de tales desarrollos científicos, que vayan más allá del debate bioético ${ }^{30}$.

Los elementos concernidos a partir de las tendencias aquí apuntadas son de hondo calado sociológico, en la medida que los avances en este terreno están quebrantando supuestos admitidos hasta hoy, a la par que nos adentramos en esferas que nunca habían sido franqueadas. El hombre, siguiendo a Jacques Testart (Testart, 1988), ha devenido en un ser "transparente" y susceptible de manipulación desde perspectivas eugenésicas.

En resumen, nos encontramos ante la perspectiva de una nueva era de la biotecnología y de la Reprogenética, que ya está conllevando notables procesos de cambio en el ritmo de la historia. La Sociología tiene la virtualidad de poder captar su dirección, debiendo evitar caer en las ensoñaciones de los intelectualismos y también, en ocasiones, de un esterilizante pragmatismo. Lo que en buena medida procede es analizar y explicar los objetivos y efectos de estas innovaciones, puesto que pueden llegar a situarse por encima de la voluntad, el deseo y la intención de los individuos particulares.

Por ello, la cuestión sociológica de fondo que se intenta plantear en este texto es que los nuevos enfoques científicos, surgidos al hilo de los desarrollos y avances de la Reprogenética, cambiarán los valores y esquemas sociales actualmente establecidos. Basta para ello con recordar las palabras del astrónomo Fred Hoyle, quien afirmó hace más de treinta años que: "... dentro de veinte años, los físicos nucleares, que sólo fabrican inofensivas bombas de hidrógeno trabajarán en libertad, en cambio los biólogos moleculares lo harán detrás de las alambradas eléctricas" (Lacadena, 1988: 144). 
1 En 1978 nació Louise Joy Brown, primer ser humano concebido a partir de un procedimiento de reproducción asistida. En España tuvimos que esperar hasta el año 1984, fecha en la que vino al mundo Victoria Ana Perera, en la Clínica Dexeus de Barcelona.

2 A diferencia de la esterilidad, entendida como la incapacidad para concebir, susceptible de ser corregida mediante procedimientos quirúrgicos y la reproducción asistida, la infertilidad es una incapacidad permanente para concebir. Las causas más frecuentes de esterilidad se deben, según datos ofrecidos por la Sociedad Española de Fertilidad, a factores masculinos $(32,7 \%)$, a factores rubrico/femeninos $(26,4 \%)$ y a causas mixtas $(23,3 \%)$ (Estudio SEF, 2009).

3 Un 56\% de los encuestados en la Fundación Sistema, Encuesta sobre Tendencias Familiares-2000 consideraron que hacia el 2010 las técnicas de reproducción asistida serían más frecuentes que en el año 2000.

4 En algo más de tres décadas la edad media en la que las mujeres españolas tienen sus primeros hijos se ha elevado. Si en 1975 los tenían con 25,24 años, en 1995 con 28,39 años, y en 2010 se sitúa en el máximo histórico de 31 años.

5 Sirva de ejemplo que en el año 2006 se registró en España un 56\% menos de niños nacidos con Síndrome de Down respecto al año 1980, debido en buena medida a que el 95\% de las madres a las que se diagnosticó que su hijo padecería esta cromosopatía decidieron acudir al aborto terapéutico.

Recibido: 10 de mayo de 2011 Aceptado: 20 de junio de 2011 noce el daño patrimonial derivado de un diagnóstico prenatal erróneo que privó a los padres de un niño nacido con sindrome de Down la oportunidad de acudir al aborto terapéutico. Cada uno de los padres recibirá 75.000 euros y el niño tendrá una pensión vitalicia de 1.500 euros. Teniendo en cuenta este tipo de situaciones y que desde el año 2005 han aumentado los casos de diagnósticos imprecisos (en torno a una veintena), algunos juristas plantean necesario que se regule sobre esta cuestión, evitando así inseguridades jurídicas y estandarizar las indemnizaciones.

7 Así se contempla en el artículo 5 de la Ley 14/2006, según el cual "la donación será anónima y deberá garantizarse la confidencialidad de los datos de identidad de los donantes por los bancos de gametos, así como, en su caso, por los registros de donantes y de actividad de los centros que se constituyan. Los hijos nacidos tienen derecho por sí o por sus representantes legales a obtener información general de los donantes que no incluya su identidad...".

8 Según el artículo 5.5 de la Ley 14/2006 "... Sólo excepcionalmente, en circunstancias extraordinarias que comporten un peligro cierto para la vida o la salud del hijo o cuando proceda con arreglo a las Leyes procesales penales, podrá revelarse la identidad de los donantes, siempre que dicha revelación sea indispensable para evitar el peligro o para conseguir el fin legal propuesto. Dicha revelación tendrá carácter restringido y no implicará en ningún caso publicidad de la identidad de los donantes".

9 La demografía dinámica estudia el comportamiento o los movimientos intrínsecos y extrínsecos de las poblaciones humanas, desde la perspec- 
tiva de su evolución en el transcurso del tiempo y los diversos mecanismos por los que se modifica la dimensión, estructura y distribución geográfica de dichas poblaciones.

10 Esta idea es uno de los principales resultados de una investigación realizada por RAND Europa y presentada por primera vez en el Congreso de la Sociedad Europea para la Reproducción Asistida y Embriología del año 2006. Además, se concluyó que las técnicas reproductivas tienen capacidad para estimular la fertilidad en Europa y su uso podría compararse a las políticas demográficas utilizadas por los diversos gobiernos para potenciar la natalidad.

11 La Ley 14/2006, de 26 de mayo, sobre Técnicas de Reproducción Humana Asistida actual no recoge explícitamente estos casos, si bien regula que "1. Las técnicas de reproducción asistida se realizarán solamente cuando haya posibilidades razonables de éxito, no supongan riesgo grave para la salud, física o psíquica, de la mujer - la posible descendencia y previa aceptación libre y consciente de su aplicación por parte de la mujer, que deberá haber sido anterior y debidamente informada de sus posibilidades de éxito, así como de sus riesgos y de las condiciones de dicha aplicación" (Art. 3.1). Además, en el Artículo 6.2 se dice que "Entre la información proporcionada a la mujer, de manera previa a la firma de su consentimiento, para la aplicación de estas técnicas se incluirá, en todo caso, la de los posibles riesgos, para ella misma durante el tratamiento y el embarazo y para la descendencia, que se puedan derivar de la maternidad a una edad clínicamente inadecuada", pudiendo dar lugar a diversas interpretaciones sobre qué se entiende por edad clínicamente inadecuada. 2. En el caso de la fecundación in vitro y técnicas afines".

12 Según Naciones Unidas si para el período temporal 2010-2015 la tasa de fertilidad se estabilizará en los países más desarrollados en 1,64 hijos por mujer, entre los años 2030-2035 ascenderá ligeramente a 1,70 y entre el año 2045 y 2050 alcanzará 1,73 (ONU, 2008).

13 La Ley 14/2006, de 26 de mayo, sobre Técnicas de Reproducción Humana Asistida, dice que se podrá "... practicar técnicas de diagnóstico preimplantacional para:

a) La detección de enfermedades hereditarias graves, de aparición precoz y no susceptibles de tratamiento curativo posnatal con arreglo a los conocimientos cientificos actuales, con objeto de llevar a cabo la selección embrionaria de los preembriones no afectos para su transferencia.

b) La detección de otras alteraciones que puedan comprometer la viabilidad del preembrión..."

14 El más conocido la amniocentesis, existiendo otras pruebas como la biopsia de blastómero, la biopsia de tejido extraembrionario o la biopsia de cuerpo polar.

15 El diagnóstico genético preimplantacional se justifica en la Ley en los siguientes términos: "... El diagnóstico genético preimplantacional abre nuevas vías en la prevención de enfermedades genéticas que en la actualidad carecen de tratamiento y a la posibilidad de seleccionar preembriones para que, en determinados casos y bajo el debido control y autorización administrativos, puedan servir de ayuda para salvar la vida del familiar enfermo" (Exposición de Motivos, Ley 14/2006 sobre Técnicas de Reproducción Humana Asistida 14/2006).

16 La Comisión Nacional de Reproducción Humana Asistida fue creada a instancias del Real Decreto 415/1997, de 21 de marzo, en donde se dice: "Se crea la Comisión Nacional de Reproducción Humana Asistida, como un órgano colegiado de carácter permanente y consultivo, dependiente del Ministerio de Sanidad y Consumo, adscrito a la Secretaría General de Sanidad, dirigido a orientar acerca de la utilización de las técnicas de reproducción asistida y colaborar con las Administraciones públicas sanitarias en lo relativo a dichas técnicas y sus derivaciones científicas..." El Decreto previó, en su Artículo 4, además de funciones de asesoramiento, la emisión de informes, entre ellos los "proyectos de investigación y experimentación con gametos, preembriones y fetos humanos". Disposición mantenida en la Ley14/2006, sobre Técnicas de Reproducción Humana Asistida, a la que se le ha añadido la elaboración de informes, que se especifica son "preceptivos" en diferentes casos, incluidos algunos de diagnóstico genético preimplantacional.

17 Según la Ley 14/2006, de 26 de mayo, sobre Técnicas de Reproducción Humana Asistida "... se entiende por preembrión el embrión in vitro constituido por el grupo de células resultantes de la división progresiva del ovocito desde que es fecundado hasta 14 días más tarde" (Capítulo I. Artículo 1.2).

$18 \mathrm{El}$ diagnóstico genético prenatal consiste en un screening o criba de enfermedades genéticas en el feto, realizado a partir de pruebas como la amniocentesis o la biopsia de las vellosidades crónicas. 
19 Actualmente en algunas zonas del mundo como India y China se utiliza el aborto selectivo a favor de nacimientos masculinos. Un estudio publicado en la revista The Lancet en el año 2006, con datos del año 1998, puso de manifiesto que sólo en India el aborto selectivo a favor de los varones había generado un desequilibrio entre sexos de $10 \mathrm{mi}-$ llones menos de mujeres. Según el informe Estructura Social de la China Contemporánea, de la Academia de Ciencias Sociales (CASS), publicado en enero de 2010, en el año 2005 nacieron 119 hombres por cada 100 mujeres debido a los abortos selectivos de fetos femeninos. Se trata del principal problema que está afectando su estructura demográfica. De seguir esta tendencia de evolución en 2020 habrá unos 24 millones de solteros, en el que con toda seguridad seguirá siendo el país con más población del mundo.

20 Según el I Informe Anual de la Comisión Nacional de Reproducción Humana Asistida del año 1998 se debe entender por clonación no reproductiva "... la aplicación de técnicas de clonación en cultivos celulares o en preembriones preimplantatorios sin intención de producir un individuo clónico vivo, sino con objeto de establecer cultivos de tejidos -y si fuera posible, de órganos- a partir de células troncales del embrión o células ser, que son células inmaduras con capacidad de auto regeneración y diferenciación. Tales cultivos pueden ser establecidos con fines de investigación básica o clínica en la reparación de tejidos u órganos dañados, en cuyo caso algunos la denominan clonación terapéutica".

21 Para Lee M. Sil ver los desarrollos futuros en reproducción asistida e in- geniería genética en humanos puede conducir a un nuevo modelo de sociedad caracterizado por una extrema polarización social, con la emergencia de dos clases sociales contrapuestas: una integrada por "humanos naturales" y otra conformada por "humanos gen-enriquecidos" o "gen-ricos" (Lee M. Silver, 1998).

22 Así se contempla en el Artículo 5 de la Ley 14/2006 según el cual "la donación será anónima y deberá garantizarse la confidencialidad de los datos de identidad de los donantes por los bancos de gametos, así como, en su caso, por los registros de donantes y de actividad de los centros que se constituyan. Los hijos nacidos tienen derecho por sí o por sus representantes legales a obtener información general de los donantes que no incluya su identidad... Sólo excepcionalmente, en circunstancias extraordinarias que comporten un peligro cierto para la vida o la salud del hijo o cuando proceda con arreglo a las Leyes procesales penales, podrá revelarse la identidad de los donantes, siempre que dicha revelación sea indispensable para evitar el peligro o para conseguir el fin legal propuesto. Dicha revelación tendrá carácter restringido y no implicará en ningún caso publicidad de la identidad de los donantes"

23 Véase artículo 6.1 de la Ley 14/2006 según el cual "En la aplicación de la técnicas de reproducción humana asistida, la elección del donante de semen solo podrá realizarse por el equipo médico que aplica la técnica, que deberá preservar las condiciones de anonimato de la donación. En ningún caso podrá seleccionarse personalmente el donante a petición de la receptora. En todo caso, el equipo médico correspondiente deberá pro- curar garantizar la mayor similitud fenotípica e inmunológica posible de las muestras disponibles con la mujer receptora".

24 En este caso la Ley 14/2006 prevé en su artículo 6.3 que "Si la mujer estuviera casada se precisará, además, el consentimiento de su marido, a menos que estuvieran separados legalmente o de hecho y así conste de manera fehaciente. El consentimiento del cónyuge, prestado antes de la utilización de las técnicas, deberá reunir idénticos requisitos de expresión libre, consciente y formal".

25 En octubre de 2006 en España, dos lesbianas lograron ser reconocidas como madres de un bebé gestado por inseminación artificial, sin necesidad de realizar un trámite de adopción, tal como establece la ley.

26 La legislación española ha resuelto este tema, al sustituir la expresión padre por progenitor A y la expresión madre por progenitor B. Estos cambios han sido introducidos tras la entrada en vigor de la Ley de matrimonios homosexuales. En la Orden/JUS/568/2006, de 8 de febrero, publicada el 3 de marzo en el BOE, sobre modificación de modelos de acuerdos y certificaciones del Registro Civil y del Libro de Familia. Además, se contempla para las parejas formadas por personas del mismo sexo emplear la terminología cónyuge $A$ y cónyuge $B$.

27 La Ley 14/2006 sobre Técnicas de Reproducción Humana Asistida es clara sobre el tema pues considera infracción muy grave "la práctica de técnicas de transferencia nuclear con fines reproductivos" (artículo 26.C.9. $)^{\text {). }}$

28 El ginecólogo italiano Severino Antinazi, "padre médico" de los niños italianos nacidos de madres con más 
de sesenta años, es uno de los más firmes defensores de este tipo de clonación.

29 De igual modo que con otras técnicas desde la Bioética y el Bioderecho se reclama la proclamación de un "derecho a ser fruto del azar" y de un "derecho a la ignorancia", es decir, a no saber o creer saber de uno mismo anticipadamente.

30 La Real Academia Española define a la Bioética como la "Aplicación de la ética a las ciencias de la vida".

\section{BIBLIOGRAFÍA}

Comisión Especial de Estudio sobre Fertilización Extracorpórea, Madrid, Sesión del 23 de septiembre de 1985.

Durkheim, Emile (1973): De la división del trabajo social, Editorial Shapire, Buenos Aires, p. 153.

Estudio Sociedad Española Fertilidad (2009): Registro de Inseminaciones (IAC-IAC) de la SEF. Año 2006.
Estudio Sociedad Española Infertilidad (2010): Registro de Inseminaciones (IAC-IAC) de la SEF, Año 2008.

Fundación Sistema (2000): Encuesta sobre Tendencias Familiares-2000.

GETS (1997): Estudio Delphi sobre tendencias científico-tecnológicas en España, Editorial Sistema, Madrid.

GETS (2002): Estudio Delphi sobre tendencias científico-tecnológicas, 2002, Editorial Sistema, Madrid.

GETS (2005): Estudio Delphi sobre Ingeniería Genética y Biotecnología, Material de trabajo, Fundación Sistema, Madrid.

Heritier, Francoise (1996): Masculin/Féminin. La Pensée de la Différence, Odile Jacob, Paris.

INE (varios años): Indicadores demográficos básicos. http://www.ine.es/jaxi/ menu.do? type $=$ pcaxis $\&$ path $=/$ t20/ p318/idbetfile $=$ pcaxis.

INE (varios años): Movimiento Natural de la Población, varios años.

Lacadena, José Ramón (1988): Fundamentación de la Bioética y manipulación genética, Universidad Pontificia de Comillas, Madrid.

Ley 14/2006, de 26 de mayo, sobre Técnicas de Reproducción Humana Asistida.

Ministerio de Sanidad y Consumo (1998): I Informe Anual de la Comisión Nacional de Reproducción Humana Asistida.

Silver Lee M. (1998): Vuelta al edén. Más allá de la clonación en un mundo feliz, Taurus.

Sánchez Morales, M. ${ }^{a}$ Rosario (2001): Las familias españolas del futuro, Sistema, Madrid.

Simmel, George (1977): "La autoconservación de los grupos" en Estudios sobre las formas de socialización, Biblioteca de la Revista de Occidente, Madrid, p. 529.

Testart, Jacques (1988): El embrión transparente, Granica, Barcelona.

Tonnies, Ferdinand (1979): Comunidad y sociedad, Península, pp. 39-40.

UNICEF, Convención sobre los Derechos del Niño.

ONU (2008): http://www.un.org/esa/population/unpop.htm. 